\title{
Cuidado do enfermeiro na prevenção de hospitalizações infantis por condições sensíveis à atenção primária
}

Nurse care in the prevention of child hospitalizations for conditions sensitive to primary care

Atención de enfermería en la prevención de hospitalizaciones infantiles por patologías sensibles a la atención primaria

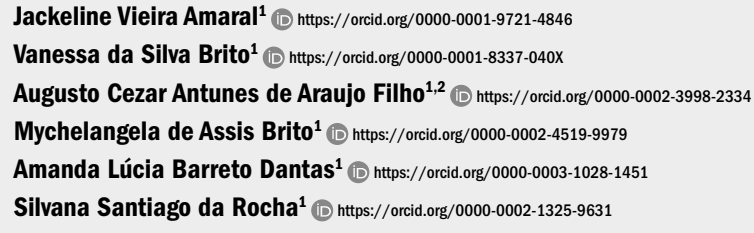

\section{Resumo}

Objetivo: Conhecer o cuidado do enfermeiro na prevenção de hospitalizações infantis por Condições Sensíveis à Atenção Primária, pelos diagnósticos de gastroenterite, pneumonia e asma.

Métodos: Pesquisa descritiva de abordagem qualitativa, realizada na cidade de Teresina nos meses de janeiro e fevereiro de 2021. Participaram do estudo 11 enfermeiros. A coleta baseou-se em entrevista semiestruturada. Os dados foram analisados mediante a análise de conteúdo de Bardin. Aprovada pelo Comitê de Ética em pesquisa da Fundação Municipal de Saúde e da Universidade Federal do Piauí, segundo o parecer $n^{0}$ 4.501.548.

Resultados: Orientações de higiene, aleitamento materno, imunização, limpeza domiciliar e evitar exposição à alérgenos. Criança com alguma condição: consulta de enfermagem e encaminhamento ao médico.

Conclusão: Os cuidados contemplam os manuais do Ministério da Saúde e protocolos da Fundação Municipal de Saúde de Teresina. Ressalta-se a importância de políticas públicas, melhor gestão dos recursos das Unidades Básicas de Saúde e educação continuada.

\section{Abstract}

Objective: To understand the care of nurses in the prevention of hospitalizations for children due to Conditions Sensitive to Primary Care, by diagnoses of gastroenteritis, pneumonia and asthma.

Methods: Descriptive research with a qualitative approach, carried out in the city of Teresina in January and February 2021. Eleven nurses have participated in the study. The collection was based on a semi-structured interview. Data were analyzed using Bardin's content analysis. Approved by the Research Ethics Committee of the Municipal Health Foundation and the Federal University of Piauí, according to opinion $n^{\circ} 4.501 .548$.

Results: Hygiene guidelines, breastfeeding, immunization, household cleaning and avoiding exposure to allergens. Child with any condition: nursing consultation and referral to the doctor.

Conclusion: The care includes manuals from the Ministry of Health and protocols from the Municipal Health Foundation of Teresina. It emphasizes the importance of public policies, better management of the resources of Basic Health Units and continuing education.

\section{Resumen}

Objetivo: Comprender el cuidado del enfermero en la prevención de hospitalizaciones de niños por Condiciones Sensibles a la Atención Primaria, mediante diagnósticos de gastroenteritis, neumonía y asma.

Métodos: Investigación descriptiva con enfoque cualitativo, realizada en la ciudad de Teresina en enero y febrero de 2021. Once enfermeras participaron en el estudio. La recopilación se basó en una entrevista semiestructurada. Los datos se analizaron mediante el análisis de contenido de Bardin. Aprobado por el Comité de Ética en Investigación de la Fundación Municipal de Salud y la Universidad Federal de Piauí, según dictamen nº 4.501.548.

Resultados: Pautas de higiene, lactancia, inmunización, limpieza del hogar y evitar la exposición a alérgenos. Niño con cualquier condición: consulta de enfermería y derivación al médico.

Conclusión: La atención incluye manuales del Ministerio de Salud y protocolos de la Fundación Municipal de Salud de Teresina. Destaca la importancia de las políticas públicas, una mejor gestión de los recursos de las Unidades Básicas de Salud y la educación continua.

\section{Como citar:}

Amaral JV, Brito VS, Araújo Filho AC, Brito MA, Dantas AL, Rocha SS. Cuidado do enfermeiro na prevenção de hospitalizações infantis por condições sensíveis à atenção primária. Rev Soc Bras Enferm Ped. 2021;21(2):110-8.
Descritores

Atenção primária à saúde; Hospitalização; Criança; Enfermagem pediátrica

Keywords

Primary health care; Hospitalization; Child; Pediatric nursing

\section{Descriptores}

Atención primaria de salud; Hospitalización; Niño; Enfermería pediátrica

\footnotetext{
${ }^{1}$ Universidade Federal do Piauí, Teresina, PI, Brasil.

${ }^{2}$ Universidade Estadual do Piauí, Teresina, PI, Brasil.

Conflitos de interesse: nada a declarar.

Submetido: 26 de Julho de 2021 | Aceito: 3 de Dezembro de 2021

Autora correspondente: Jackeline Vieira Amaral | E-mail: jackelinevamara@@gmail.com

Dol: http://dx.doi.org/10.31508/1676-379320210016
} 


\section{Introdução}

Na Atenção Primária à Saúde (APS), a implementação da Estratégia Saúde da Família (ESF) têm possibilitado uma ampliação da capacidade resolutiva, evitando agravos e, consequentemente, a necessidade de encaminhamento para a atenção secundária ou terciária. ${ }^{(1)}$ No âmbito da saúde da criança, a ESF proporciona a aproximação desse grupo com os serviços primários, sendo responsável por acompanhar o crescimento e desenvolvimento infantil, assim como promover, ações de saúde voltadas ao estímulo da amamentação e imunização, em prol da redução da taxa de morbimortalidade infantil. (2)

As crianças necessitam de cuidados que as contemplem em sua integralidade. ${ }^{(3)} \mathrm{Na}$ oferta desse cuidado, o enfermeiro é visto como importante instrumento, intervindo diante de fatores de risco e desenvolvendo cuidados para a prevenção de doenças e promoção da saúde. ${ }^{(4)}$ Ademais, a atenção à saúde nesses moldes é resultante da focalização em aspectos familiares e comunitários, o que fortalece o vínculo com os serviços de saúde e permite o conhecimento da realidade em que a criança está inserida. ${ }^{(3,5)}$

Nessa perspectiva, a APS dispõe de princípios essenciais que fundamentam a prestação de serviços direcionados para a prevenção dos diagnósticos das Condições Sensíveis à Atenção Primária (CSAP), listadas na portaria do Ministério da Saúde (MS) no 221, de 17 de abril de 2008. ${ }^{(5)}$ As CSAP são doenças em que os serviços primários podem prevenir, identificar e tratar precocemente, evitando hospitalizações desnecessárias. Desse modo, a taxa de hospitalizações por CSAP corresponde a um indicador de qualidade e acesso dos serviços primários de sáude. ${ }^{(5,6)}$

Nos últimos anos, com o desenvolvimento de políticas, programas e ações direcionados para a redução da morbimortalidade infantil, obteve-se o declínio da taxa de hospitalizações evitáveis pela APS. No Brasil, foi observada uma redução de $45 \%$ nas taxas de internações por CSAP, no período de 2001 a 2016.(7) No entanto, o Nordeste brasileiro é uma das regiões que apresenta a maior taxa de hospitalizações por CSAP, correspondendo a uma média anual de $427,14 / 10^{4}$ hospitalizações de crianças menores de cinco anos de 2004 a 2013, o que representa uma taxa maior que a nacional, tendo em vista que esta corresponde a 358,56/10 no mesmo período. ${ }^{(8)}$
Concomitante a situação, o Estado do Piauí possui taxas maiores que a região Nordeste, alcançando média de $478,53 / 10^{4}$ hospitalizações de crianças menores de cinco anos, no período de 2004 a 2013. ${ }^{(8)}$ Destaca-se que as gastroenterites, pneumonias e asma ainda são responsáveis pela maior parte dessas internações, na capital do Estado, alinhando-se a fatores socioeconômicos e demográficos. ${ }^{(9)}$

Nesse contexto, destaca-se que a atenção precoce no nível primário é entendida como forma eficaz para prevenir e evitar a evolução dessas doenças. Desse modo, elevadas taxas de hospitalizações por CSAP remetem a necessidade de conhecer sobre os cuidados destinados à prevenção das hospitalizações pelas condições predominantes. Além disso, como o enfermeiro está envolvido nos principais eixos de cuidados à criança no nível primário, é imprescindível o conhecimento da atuação desse profissional, com relação às ações que busquem proporcionar melhorias de saúde da população e, consequentemente, redução da morbimortalidade. ${ }^{(10)}$

Nesse sentido, esta pesquisa tem como objetivo conhecer o cuidado do enfermeiro na prevenção de hospitalizações infantis por Condições Sensíveis à Atenção Primária, pelos diagnósticos de gastroenterite, pneumonia e asma. Assim, para a compreensão da contribuição do enfermeiro, elaborou-se a seguinte questão norteadora: "Como está sendo desenvolvido o cuidado do enfermeiro na prevenção das hospitalizações infantis por Condições Sensíveis à Atenção Primária?".

\section{Métodos}

Trata-se de uma pesquisa descritiva de abordagem qualitativa.

A pesquisa foi realizada na cidade de Teresina-PI, nos meses de janeiro e fevereiro de 2021. A capital dispõe de quatro regionais de saúde (Centro/Norte, Sul, Leste e Sudeste), nas quais estão distribuídas 95 Unidades Básicas de Saúde (UBS), sendo 81 delas localizadas na zona urbana.

Para a presente pesquisa foram consideradas as UBS da zona urbana, visto a dificuldade de comunicação online com profissionais da zona rural. Ressalta-se que na elaboração da metodologia foi utilizado o instrumento Consolidated Criteria for Reporting Qualitative Research (COREQ)..$^{(11)}$ 
Inicialmente, foram sorteadas 20 UBS, sendo cinco de cada regional de saúde. Participaram deste estudo 11 enfermeiros que estavam atuando diretamente nas atividades assistenciais da ESF. Os enfermeiros os quais não estavam trabalhando, por motivos como férias, afastamento por doença, acidente de trabalho ou gestação, e aqueles que não executavam atividades diretamente com o público infantil, como estar restrito a atividades administrativas, foram eliminados da pesquisa. Utilizou-se a saturação teórica dos dados como critério para interromper a inserção de novos participantes. ${ }^{(12)}$

Para a coleta dos dados, as UBS sorteadas foram informadas por contato telefônico e receberam por e-mail a autorização dos Comitês de Ética em Pesquisa (CEP), posteriormente repassaram endereço eletrônico dos enfermeiros, os quais foram instruídos quanto a pesquisa e receberam um link do Google Forms com o Termo de Consentimento Livre e Esclarecido (TCLE) e um questionário. O questionário abordou dados sociodemográficos e profissionais, e permitiu agendamento de data e horário para a entrevista.

Destaca-se que, com o propósito de garantir a segurança dos pesquisadores e participantes frente ao atual cenário de pandemia do novo coronavírus, as entrevistas foram realizadas por meio do serviço de comunicação por vídeo, o Google Meet.

A coleta aconteceu por meio de um roteiro de entrevista semiestruturado. Previamente, ocorreu a avaliação do roteiro construído, por meio do pré-teste com dois enfermeiros, os quais não foram incluídos na população do estudo. Após isso, foram realizadas alterações no roteiro, a fim de aperfeiçoá-lo. Contou-se com perguntas abertas, as quais voltaram-se para as condições predominantes no município, gastroenterite, pneumonia e asma..$^{(9)}$ Assim, o roteiro constituiu-se pelas perguntas: “Quais os cuidados que você realiza com as crianças da sua área adstrita direcionados para a prevenção das gastroenterites, pneumonia e asma?", "Quais as condutas aos pacientes com gastroenterites, pneumonia e asma?", "Quais as dificuldades para prevenir condições sensíveis à atenção primária?" e "Quais os facilitadores para prevenir condições sensíveis à atenção primária?".

As entrevistas foram gravadas, em voz e imagem, conforme autorização precedente dos participantes por meio de sua assinatura eletrônica do TCLE, via Google Forms. A duração das entrevistas variou entre
10 a 30 minutos. A gravação ocorreu com auxílio de ferramenta do próprio Google Meet, sendo que ao concluir a coleta, foi realizado download dos dados para dispositivo eletrônico local e, subsequentemente, foram excluídos do Google Drive. Após todos esses procedimentos, as entrevistas foram transcritas utilizando o Microsoft Word ${ }^{\circledR} \mathrm{e}$, em seguida, foram enviadas aos participantes, para a realização da validação.

Ressalta-se que foram priorizados ambientes silenciosos e isolados, restritos apenas ao enfermeiro e às pesquisadoras, preservando a confidencialidade das informações coletadas. Para garantir o anonimato dos participantes, os enfermeiros foram identificados com os seguintes nomes de flores: íris, girassol, hibisco, lírio, magnólia, jacinto, camélia, lótus, margarida, tulipa e orquídea.

A análise dos dados baseou-se na análise de conteúdo de Bardin. ${ }^{(13)} \mathrm{A}$ abordagem consiste em um conjunto de técnicas de análise das comunicações com o propósito de obter, por procedimentos, sistemáticos e objetivos, de descrição do conteúdo das mensagens, a compreensão e o alcance de deduções lógicas e justificadas quanto aos aspectos referentes à origem da mensagem. Essa técnica é constituída por três etapas, sendo elas a pré-análise, exploração do material e tratamento dos resultados. ${ }^{(13)}$

Na primeira etapa, a pré-análise, o material foi organizado com o propósito de possibilitar uma visão do conjunto. Para isso foi realizada a leitura flutuante de todo o material e logo em seguida formulou-se o corpus. Ainda nessa etapa, recortes do texto foram feitos, selecionando índices e estabelecendo indicadores, o que auxiliou nas etapas seguintes.

$\mathrm{Na}$ exploração do material as decisões tomadas na primeira etapa foram implementadas seguidas por codificação e categorização dos dados. Na etapa de codificação as unidades de registros (frases) foram demarcadas e em seguida agregadas em categorias. Após isso, prosseguiu-se com a etapa de tratamento dos resultados. Nessa última etapa, desenvolveu-se inferências e interpretações acerca do conteúdo.

A pesquisa ocorreu em consonância com as resoluções no 466/2012 e no 510/2016, e com a carta circular no 1/2021 do Conselho Nacional de Saúde, sendo aprovada pelo Comitê de Ética em pesquisa da Fundação Municipal de Saúde (FMS) e da Universidade Federal do Piauí, segundo o parecer no 4.501 .548 . 


\section{Resultados}

Dos 11 enfermeiros entrevistados, nove eram do sexo feminino. No que se refere a cor/raça, mais da metade dos participantes se autodeclararam brancos. A idade variou entre 25 e 59 anos, e no que concerne ao tempo que finalizou a graduação, variou entre três e 35 anos. Em relação ao tempo de trabalho na ESF, houve variação entre um e 24 anos. Do total, 10 profissionais enfermeiros possuíam curso de pós-graduação lato sensu, sendo que cinco dos entrevistados possuíam especialização em Saúde da Família. Quando questionados sobre treinamento na área de Saúde da Criança, seis entrevistados disseram que nunca receberam treinamento específico.

Os enfermeiros com maior tempo de trabalho na ESF e aqueles que possuíam especialização em ESF forneceram relatos mais detalhados quanto aos cuidados na prevenção das hospitalizações por CSAP. Destaca-se que aqueles com especialização em ESF relataram com mais propriedade e segurança o desenvolvimento de ações pontuais para evitar e tratar as doenças estudadas.

Após a análise completa dos conteúdos descritos nas falas dos enfermeiros foi possível agrupá-los nas seguintes categorias: "Cuidados direcionados à prevenção das condições sensíveis à atenção primária"; "Conduta do enfermeiro aos pacientes com gastroenterite, pneumonia e asma para evitar a hospitalização"; "Dificuldades enfrentadas para a prevenção das hospitalizações por condições sensíveis à atenção primária" e "Facilitadores para a prevenção das hospitalizações por condições sensíveis à atenção primária".

\section{Cuidados direcionados à prevenção das condições sensiveis à atenção primária}

A oferta do cuidado integral à criança prestado durante qualquer consulta realizada na UBS, considerando o indivíduo como um todo, desde o acompanhamento do crescimento, a identificação da presença de alterações e conhecimento de suas vulnerabilidades, é mostrado como passo inicial para a prevenção das CSAP.

A prevenção parte da avaliação de enfermagem periódica, seja por demanda espontânea ou por consulta de rotina [...], busca ativa, conhecimento da área [...] de condições de vulnerabilidade e exposição a determinados fatores de risco (Lótus).
[...] Quando a criança chega no atendimento, como o atendimento para o bolsa família, a gente já considera como um todo, vê o peso, estatura, imunização, presença de prurido, alguma dermatite, certo? A gente tem que ver assim, a criança como um todo [...] (Jacinto).

A prevenção das CSAP tem início com a consulta de pré-natal, na qual são realizadas condutas com o propósito de prevenir problemas considerados evitáveis. Subsequente ao pré-natal prossegue-se com as consultas de puericultura da criança, correspondendo ao momento em que são repassados ao responsável os primeiros cuidados que devem ter com a criança para evitar o surgimento de morbidades.

O primeiro cuidado que temos é já no pré-natal (Margarida).

Fazemos rotineiramente o acompanhamento de pré-natal, que já previne algum problema na criança, e quando a criança nasce, o que nós fazemos na nossa rotina é uma visita domiciliar à puérpera e ao RN na primeira semana, né? Faz exame físico da criança, vê amamentação como é que tá, zêe a questão das vacinas [...] (Íris).

As medidas para a prevenção de gastroenterites, uma das doenças responsáveis pela maior parte das hospitalizações por CSAP no município de Teresina, consistem em orientações destinadas, principalmente, à amamentação exclusiva, cuidados com a higiene de alimentos e tratamento da água. Foram ressaltadas também as orientações quanto à introdução alimentar saudável sem alimentos industrializados e a vacina contra o rotavírus.

Para prevenção das gastroenterites [...] consumo de água filtrada ou fervida, vacina rotavírus em dia, explicar sobre os alimentos saudáveis e não saudáveis (Tulipa).

A gente sempre orienta, principalmente, com relação à amamentação exclusiva até o sexto mês. Se a mãe por algum motivo vai retornar ao trabalho, vai ter que introduzir outro alimento antes dos 6 meses, a gente reforça os cuidados de higiene, da nutrição, dos alimentos corretos [...] (Margarida).

[..] A gente vem trabalhando com nossos agentes de saúde na promoção de saúde dessas crianças, como por exemplo distribuição de hipoclorito [de sódio] para tentar reduzir as doenças diarreicas (Lírio). 
Além das atividades realizadas nas UBS para a prevenção de gastroenterites, o Programa de Saúde na Escola possibilita desenvolvimento do cuidado em ambiente diferente. Esse cuidado consiste na distribuição de vermífugos às crianças e realização de orientações de higiene.

Tem o programa da geo-helmintíase, no qual é feita a aplicação de vermífugos nos escolares acima de 5 anos, e nos menores de 5 anos a gente já reforça a orientação da higiene na escola, como lavagem das mãos, do cuidado com unhas, de não colocar as mãos sujas na boca, então todas essas orientações a gente reforça nas escolas e creches (Margarida).

A implementação de ações destinadas à prevenção de pneumonias foi citada por poucos entrevistados, sendo mencionado com mais frequência a vacina como forma de prevenção.

Com relação a pneumonia, reforçamos as vacinas, a pneumo, que é feita no primeiro ano de vida e a contra o vírus influenza que previne várias infecções respiratórias (Margarida)

Os cuidados para asma consistem em orientações quanto às medidas preventivas para sibilância, como medidas higiênicas, com o propósito de evitar contato com alérgenos como poeira, mofo e fumaças, dando ênfase também ao aleitamento materno e a hidratação da criança.

Orientações quanto aos cuidados com o ambiente domiciliar, a fim de evitar poeira, mofo e fumaça (Lótus). A questão mesmo da limpeza, estar orientando as mães para retirar objetos que acumulem poeira, ursinhos de pelúcia, tapete e cortinas, a gente faz essa orientação sobre o ambiente, sem falar a questão da hidratação e da alimentação da criança, frisando que o aleitamento materno protege contra várias doenças (Orquídea).

\section{Conduta do enfermeiro aos pacientes com gastroenterite, pneumonia $e$ asma para evitar a hospitalização}

A consulta de enfermagem constitui-se como passo inicial para aplicação do processo de enfermagem, além de funcionar como norteadora dos cuidados necessários por toda a equipe multiprofissional, fornecendo, assim, uma assistência integral ao usuário.

Anamnese, exame físico, verificar os sinais vitais, realizar diagnóstico de enfermagem, prescrição de medicamentos de acordo com o protocolo, realizar orientações direcionadas para os problemas de saúde e, se necessário, encaminhar para consulta médica (Tulipa).

$\mathrm{Na}$ assistência à criança, inserir a mãe como protagonista do cuidado ao filho contribui para a definição da conduta pelo profissional, em especial, para o seguimento do cuidado no domicílio.

Às vezes, alguns profissionais menosprezam o que a mãe fala, mas devemos saber reconhecer a devida importância do relato da mãe pra gente direcionar nossas condutas e quando a mãe relata eu levo com muito compromisso (Lírio).

O mais importante [...] é a orientação que você dá pra mãe do que é o sinal de agravamento do quadro, para que ela não fique com a criança em casa, caso ela piore (Íris).

Os procedimentos adotados para tratar gastroenterites dependem da avaliação realizada pelo enfermeiro no momento da consulta com auxílio dos relatos da mãe. Essa avaliação direciona qual plano do manejo de gastroenterites deve ser seguido, e, consequentemente, o que deverá ser prescrito para o paciente.

Criança com diarreia a gente vai logo observar aspecto das fezes e vai investigar com a mãe ou quem tiver acompanhando, quanto tempo está com diarreia? Qual éo aspecto das fezes? [...] se é menor que seis meses, se está mamando [...], se for conduta de enfermagem, a gente já direciona pra um plano A que é só soro oral (Íris).

Com uma diarreia inicial, a gente prescreve o soro, dá as orientações direitinho, se a criança tem febre, a gente pode prescrever um antitérmico [...] orientar de não deixar de amamentar a criança, e prescrever antiemético também (Hibisco).

No tratamento da pneumonia a figura médica é citada com mais frequência, mesmo que algumas condutas possam ser realizadas pelo enfermeiro, como a prescrição da terapêutica medicamentosa para essa 
doença apresentada no manual Atenção Integrada às Doenças Prevalentes na Infância (AIDPI).

Se a criança tá muito cansada, se está respirando acima daquele ponto de corte (...) acima de 40 ou 50 [...], se tiver algum sugestivo de pneumonia automaticamente a gente encaminha para o médico da nossa equipe [...] se for um caso muito grave, encaminha para a urgência mais próxima (Lírio).

Criança chega com tosse, sibilos [...] a gente faz o exame clínico dela e vê se tem alguma crepitação e o doutor prescreve a medicação [...] (Jacinto).

As condutas para asma consistem na avaliação inicial da criança, encaminhamento ao médico para prescrição de medicamentos e, na maioria das vezes, a criança é referenciada para outros níveis de atenção.

Eu faço o primeiro atendimento [..] na hora da medicação eu encaminho pro médico (Jacinto).

Segue do mesmo jeito da pneumonia, a grande maioria sempre vai pro hospital [...] (Girassol).

O manual AIDPI norteia o profissional de saúde a desenvolver condutas assertivas e eficientes no tratamento dessas doenças. No entanto, a implementação das condutas baseadas no manual acontece de forma superficial.

Em relação à pneumonia a gente avalia segundo o AIDPI (Lírio).

A gente aplica em algumas situações. É um documento com um certo tempo, mas facilita a atuação do enfermeiro, principalmente nas doenças respiratórias, na triagem, naquelas perguntas, na abordagem, na anamnese, no exame físico (Magnólia).

Nem sempre a gente consegue fazer o plano de acordo com o protocolo de diarreia, né, [...] na maioria das vezes a gente consegue manter só com o soro oral [...] seguindo mais ou menos o protocolo do AIDPI (Íris).

\section{Dificuldades enfrentadas para a prevenção das hospitalizações por condições sensíveis à atenção primária}

A implementação das ações dos enfermeiros para a prevenção das hospitalizações por CSAP versa com dificuldades que comprometem o sucesso das medidas tomadas pelos enfermeiros. As dificuldades mencionadas foram: a baixa condição socioeconômica e de escolaridade que impedem o seguimento das orientações passadas pelo enfermeiro; a pandemia, que prejudicou a realização das visitas e atividades coletivas; a falta de treinamento e conhecimento específico destinado à prevenção de CSAP; adesão da comunidade, que influencia na consolidação das ações propostas pela equipe na área; questões estruturais, que comprometem a execução dos cuidados; e carência de recurso, que interfere na terapêutica.

A maior dificuldade é essa a questão de condições de vida da pessoa que às vezes não favorece colocarem em prática essas medidas preventivas [...] difícil orientar algumas coisas, às vezes as pessoas têm uma casa insalubre, muito fria, com animais, casa úmida, pouco ventilada, tem uma casa que é propícia a doenças respiratórias (Camélia).

Eu acho que a questão da [..] escolaridade [das mães] também influencia. (Orquídea).

Agora especificamente a COVID-19, ela nos impede por exemplo de fazer a visita domiciliar, foram suspensas atividades coletivas de educação em saúde pra evitar aglomerações [...] (Magnólia).

Acho que, no meu caso, é mesmo questão de treinamento [...] diarreia se ela agravar, eu talvez não consiga lidar, mas se houvesse capacitação, treinamento para os enfermeiros, ou mesmo eu me especializar na área, eu conseguiria resolver mais coisas, o que atrapalha é isso (Hibisco).

A grande maioria das dificuldades é pela resistência comunitária em aderir às orientações, quando se tem diarreia, por exemplo, eles mesmos já têm uma conduta (Girassol).

A cobertura vacinal aqui não é muito boa por conta da própria família que às vezes não tem muito interesse e por conta da falta de vacina também [...] aqui tem queda de energia muito frequente, é um problema muito antigo, [...] o que nos deixa sem vacina, às vezes (Íris).

Um dos problemas é que não temos medicações endovenosas na UBS, então nos casos que não conseguem ingerir nada, a gente já encaminha para o hospital [...] (Jacinto). 


\section{Facilitadores para a prevenção das hospitalizações por condições sensíveis à atenção primária}

Mesmo com as dificuldades, existem alguns facilitadores que contribuem para que seja prestada uma assistência de qualidade, como: demanda reduzida, que favorece uma atenção maior ao atendimento individual; o prontuário eletrônico, que permite conhecer o histórico do paciente e direcionar um cuidado integral; a vinculação da comunidade à ESF, que facilita o trabalho da equipe; o Agente Comunitário de Saúde (ACS), que é responsável pelo elo entre a equipe e a comunidade; e o vínculo entre profissional e família, que é determinante para que a longitudinalidade seja mantida.

[...] Mesmo o calendário do Ministério da Saúde preconizando as consultas [...] que coincidem com as vacinas, como a nossa demanda não é muito grande, a gente tem condições de fazer, todo mês, até a criança completar um ano, né? Temos hoje um instrumento no prontuário eletrônico [...], e é onde você verifica a presença de algumas patologias, o próprio sistema já lhe dá esse direcionamento [...] (Íris).

O que facilita é porque hoje em Teresina praticamente $100 \%$ da área é coberta pela estratégia [saúde da família], que facilita a equipe trabalhar [...], eu acredito que seja a vinculação da comunidade (Lírio).

A questão do agente [comunitário] de saúde é muito importante [...] ele facilita o elo entre a comunidade e a equipe (Orquídea).

Eu acho que esse vínculo com a mãe é facilitador, a confiança que ela tem com o profissional facilita muito ela voltar (Hibisco).

A nossa comunicação é extremamente íntima, conhecemos [...] a criança que foi internada, como está e se retornou [...] (Jacinto).

\section{Discussão}

A prevenção das hospitalizações infantis por CSAP envolve cuidados empregados tanto na prevenção dessas condições como no tratamento precoce. Os cuidados relatados pelos enfermeiros baseiam-se em uma assistência que considera a criança em sua integralida- de, reconhecendo os diferentes contextos em que ela está inserida e, consequentemente, suas vulnerabilidades. Com isso, entende-se que o atributo integralidade é norteador dos cuidados a serem desenvolvidos pelo enfermeiro na prevenção das hospitalizações infantis por CSAP, uma vez que promove a identificação das limitações do indivíduo e, portanto, orienta o planejamento dos cuidados.

O desenvolvimento do cuidado integral, iniciado no pré-natal e seguido pelo acompanhamento do crescimento e desenvolvimento da criança, evidencia-se nos depoimentos dos enfermeiros, como forma de minimizar as condições evitáveis que afligem esse grupo. Tal relato encontra-se em consonância com os aspectos da Política Nacional de Atenção Integral à Saúde da Criança (PNAISC), que tem como foco principal a promoção e proteção à saúde da criança e ao aleitamento materno, assegurando o acompanhamento desde a gestação. $^{(14)}$

Ao longo do acompanhamento da criança na APS, as atividades mencionadas pelos enfermeiros referentes à prevenção de gastroenterites, pneumonia e asma, voltam-se para educação em saúde. Nessa perspectiva, é dado ênfase em orientações quanto à alimentação da criança, destacando-se a necessidade do aleitamento materno exclusivo até seis meses de vida. Essa abordagem confere relevância em virtude da composição do leite materno, no qual a presença de imunoglobulina A é responsável por proporcionar proteção de mucosas digestiva e respiratória contra a entrada de microrganismos. ${ }^{(15)}$

Na prevenção das gastroenterites são também realizadas recomendações voltadas à higiene e tratamento da água. Entretanto, a prevenção das hospitalizações por essa condição depende de fatores determinantes que vão além dos serviços primários de saúde. Nesse contexto, pode-se mencionar o desenvolvimento socioeconômico como fator que interfere na concentração das CSAP. ${ }^{(16)}$ Com isso, os problemas relacionados, principalmente, ao saneamento básico, são responsáveis pela disseminação dessa condição, assim como acontece em outras regiões do país. ${ }^{(17)}$

Os aspectos socioeconômicos influenciam também na prevenção de pneumonia e asma, uma vez que as condições de moradia e de higiene podem desencadear essas doenças. A prevenção de crises asmáticas advém do seguimento oportuno de recomendações, para isso 
são necessários recursos suficientes que permitam uma moradia livre de alérgenos e umidade e, sobretudo, possibilite condições adequadas de higiene, para que os riscos de infecções também sejam minimizados. ${ }^{(18)}$

Desse modo, para assegurar a saúde desse público, as ações desenvolvidas no campo de cuidados primários precisam considerar as necessidades básicas existentes no ambiente domiciliar, uma vez que o status socioeconômico está relacionado com as hospitalizações por CSAP. Esses aspectos mostram a necessidade de ações conjuntas envolvendo políticas públicas e gestão dos serviços de saúde, a fim de implementar ações focalizadas no suprimento de carências específicas em cada contexto familiar. ${ }^{(16)}$

Além disso, independentemente da condição socioeconômica, medidas preventivas, como a imunização, são direitos da população. Nesse sentido, observou-se na presente pesquisa que os enfermeiros mencionaram com frequência a imunização contra gripe e pneumococo, como medida para evitar infecções respiratórias e, possivelmente, o desenvolvimento de pneumonia. Nesse contexto, enfatiza-se o importante papel que o enfermeiro da ESF exerce na prática de imunização, que é indispensável para a manutenção da saúde da criança. ${ }^{(19)}$

Na prestação de cuidados à criança com gastroenterites, pneumonia ou asma, os manuais do MS e protocolos da FMS asseguram ao enfermeiro condutas como a prescrição de terapêuticas medicamentosas. Contudo, as condutas mencionadas pelo enfermeiro neste trabalho, são restritas a condições iniciais, sendo que, entre as condições estudadas, esses cuidados concentram-se mais nos casos de gastroenterites. Por outro lado, pacientes com sinais e sintomas respiratórios, indicativos de pneumonias ou asma, são, na maioria das vezes, encaminhados para avaliação médica ou para serviço hospitalar.

O manual de AIDPI é norteador das condutas para a prevenção das hospitalizações infantis por CSAP. ${ }^{(19)}$ Em contrapartida, os relatos evidenciam que a implementação das condutas conforme o manual, na maioria das vezes, não ocorre. Tal situação compromete $\mathrm{o}$ atendimento das demandas da criança, podendo refletir, posteriormente, no agravamento do quadro clínico e na hospitalização. ${ }^{(19)}$

Somado a isso, destaca-se que a ausência de educação permanente, problemas estruturais e in- disponibilidade de recursos, foram mencionados como fatores que interferem no cuidado do enfermeiro. No âmbito da APS, fatores como esses interferem na oferta do cuidado integral à saúde da criança. Sobretudo, para um serviço resolutivo é exigido que a unidade de saúde disponha apropriadamente de infraestrutura e recursos. ${ }^{(10)}$ Ademais, é necessário que o enfermeiro esteja preparado para determinar as melhores condutas. ${ }^{(19)}$

Apesar disso, os cuidados ofertados na APS passaram por mudanças ao longo dos anos, dentre elas, evidencia-se a vinculação da comunidade a ESF, o que tem motivado o relacionamento entre profissionais e família, facilitando a assistência à criança. Esse vínculo permite contemplar em longitudinalidade as necessidades, problemas e prioridades familiares, construindo o plano de cuidados centrado na criança e em seus familiares. ${ }^{(3)}$ Assim, contribui positivamente para a eficácia das ações de saúde e, consequentemente, para a redução das hospitalizações infantis por CSAP. ${ }^{(6)}$

Os diferentes aspectos discutidos nesta pesquisa possibilitam uma visão geral dos cuidados que o enfermeiro da APS oferta para evitar as hospitalizações por CSAP. Ao mesmo tempo que essas informações são explanadas, a reflexão evidencia a necessidade de conhecimentos e ações com intuito de evitar e tratar precocemente as CSAP. Desse modo, esta pesquisa contribui para a verificação dos cuidados desenvolvidos pelo enfermeiro, contemplando, também, as dificuldades e, assim, os diversos pontos em que esses cuidados precisam melhorar.

Ressalta-se, como limitação desta pesquisa, que a realização da coleta de dados de forma virtual, impossibilita a observação de outros aspectos que ajudam a construir uma pesquisa qualitativa. A dificuldade de analisar ao longo da entrevista as reações que os entrevistados expressavam com os questionamentos e a impossibilidade de visualizar as demonstrações da dinâmica correspondente ao trabalho ofertado pelos enfermeiros limitaram as interpretações.

\section{Conclusão}

Pode-se concluir que os cuidados do enfermeiro na prevenção das hospitalizações por gastroenterites, 
pneumonia e asma apoiam-se na assistência integral à criança, contemplando medidas preventivas e aquelas voltadas ao tratamento da condição instalada. Entretanto, no exercício de sua profissão o enfermeiro lida com alguns obstáculos, como a falta de treinamento e conhecimento sobre a prevenção de CSAP, observada, principalmente nos enfermeiros sem especialização em ESF. Aspectos estes, causam insegurança no profissional na decisão da conduta adequada. Além disso, a carência de recursos da unidade de saúde e a baixa condição socioeconômica da família podem comprometer a conduta do enfermeiro e o seguimento dos cuidados à criança. Desse modo, ressalta-se a importância de políticas públicas com o intuito de minimizar problemas socioeconômicos da população e proporcionar melhores condições de moradia e saneamento básico. A nível das UBS é necessário melhor gestão dos recursos para que o enfermeiro consiga realizar os cuidados de prevenção e manejo das CSAP. Ademais, concomitantemente a essas mudanças, a educação permanente deve ser apropriada pelas UBS como forma de aperfeiçoar o cuidado ofertado pelo enfermeiro e, assim, melhorar a assistência prestada à criança, com a finalidade de reduzir a morbimortalidade, sobretudo por causas evitáveis.

\section{Contribuições}

Amaral JA, Brito VS, Araujo Filho ACA, Brito MA, Dantas ALB e Rocha SS contribuíram integralmente na concepção do estudo, análise e interpretação dos dados, redação do artigo, revisão crítica relevante do conteúdo intelectual e aprovação da versão final do artigo.

\section{Referências}

1. Fausto MC, Rizzoto ML, Giovanella L, Seidl H, Bousquat A, Almeida PF, et al. 0 futuro da Atenção Primária à Saúde no Brasil. Saúde Debate. 2018;42(1):12-7.

2. Damasceno SS, Nóbrega VM, Coutinho SE, Reichert AP, Toso BR, Collet N. Saúde da criança no Brasil: orientação da rede básica à atenção primária à saúde. Ciênc Saúde Colet. 2016;21(9):2961-73.

3. ReichertAP, Leônico AB, Toso BR, Santos NC, Vaz EM, Collet N. Orientação familiar e comunitária na atenção primária à saúde da criança. Ciênc Saúde Colet. 2016;21(1):119-27.

4. Ferreira SR, Périco LA, Dias VR. A complexidade do trabalho do enfermeiro na Atenção Primária à Saúde. Rev Bras Enferm. 2018;71(Supl. 1):704-9.

5. Brasil. Ministério da Saúde. Secretaria de Atenção à Saúde. Portaria $n^{0} 221$ de 17 de abril de 2008. Brasília (DF): Ministério da Saúde; 2008 [citado 2020 Abr 20]. Disponível em: http://bvsms.saude.gov.br/bvs/saudelegis/sas/2008/prt0221_17_04_2008.html.

6. Santos LP, Castro AL, Dutra VG, Guimarães RM. Internações por condições sensíveis à atenção primária à saúde, 2008-2015: uma análise do impacto da expansão da ESF na cidade do Rio de Janeiro. Cad Saúde Colet. 2018;26(2):178-83.

7. Pinto LF, Giovanella L. Do programa à estratégia saúde da família: expansão do acesso e redução das internações por condições sensíveis à atenção básica (ICSAB). Ciênc Saúde Colet. 2018;23(6):1903-13.

8. Ribeiro MG, Araujo Filho AC, Rocha SS. Hospitalizações por condições sensíveis à atenção primária em crianças do Nordeste Brasileiro. Rev Bras Saúde Mater Infant. 2019;19(2): 499-506.

9. Amaral JV, Araujo Filho AC, Rocha SS. Hospitalizações infantis por condições sensíveis à atenção primária em cidade brasileira. Av Enferm. 2020;38(1):46-54.

10. Araujo Filho AC, Rocha SS, Gouveia MT. Possibilidades para a integralidade do cuidado da criança na atenção básica. Rev Cubana Enferm. 2019;35(1):419.

11. Souza VR, Marziale MH, Silva GT, Nascimento PL. Tradução e validação para a língua portuguesa e avaliação do guia COREQ. Acta Paul Enferm. 2021;34:eAPE02631.

12. Nascimento LC, Souza TV, Oliveira IC, Moraes JR, Aguiar RC, Silva LF. Theoretical saturation in qualitative research: an experience report in interview with schoolchildren. Rev Bras Enferm. 2018;71(1):228-33.

13. Bardin L. Análise de conteúdo. São Paulo: Edições 70; 2016.

14. Menezes LG, Ciuffo LL, Gonçalves AP, Moraes JR, Souza TV, Rodrigues EC. A criança e sua família na Atenção Primária em Saúde. Rev Enferm UFPE on line. 2019;13:e241426.

15. Palmeira P, Sampaio MS. Imunologia do leite materno. RevAssoc Med Bras. 2016;62(6):58493.

16. Chun SY, Kim W, Park EC. Disparities in avoidable hospitalization by income in South Korea: data from the National Health Insurance cohort. Eur J Public Health. 2018;29(2):1-7.

17. Aguiar KC, Cohen SC, Maciel EM, Kligerman DC. Fatores de risco para ocorrência de diarreia em crianças residentes na Ilha de Guaratiba (RJ). Saúde Debate. 2020;44(124):205-20.

18. Chong Neto HJ, Solé D, Camargos P, Rosário NA, Sarinho EC, Chong-Silva DC, et al. Diretrizes da Associação Brasileira de Alergia e Imunologia e Sociedade Brasileira de Pediatria para sibilância e asma no pré-escolar. Arq Asma Alerg Imunol. 2018;2(2):163-208.

19. Branquinho ID, Lanza FM. Saúde da criança na atenção primária: evolução das políticas brasileiras e a atuação do enfermeiro. Rev Enferm Cent-0este Min. 2018;8:e2753. 